10-28-2019

\title{
Conference Proceedings: Aurora Scientific Day 2019
}

Follow this and additional works at: https://aah.org/jpcrr

\section{Recommended Citation}

Conference proceedings: Aurora Scientific Day 2019. J Patient Cent Res Rev. 2019;6:274-87. doi: $10.17294 / 2330-0698.1734$

Published quarterly by Midwest-based health system Advocate Aurora Health and indexed in PubMed Central, the Journal of Patient-Centered Research and Reviews (JPCRR) is an open access, peer-reviewed medical journal focused on disseminating scholarly works devoted to improving patient-centered care practices, health outcomes, and the patient experience. 


\section{Conference Proceedings: Aurora Scientific Day 2019}

Select abstracts published herein were presented at the 45th annual Aurora Scientific Day research symposium, held May 22, 2019, in Milwaukee, Wisconsin. This symposium provides a forum for research projects conducted by faculty, fellows, residents, and other allied health professionals affiliated with Aurora Health Care, which is part of Advocate Aurora Health, an integrated health system with hospitals and clinics in Wisconsin and Illinois.

\section{ORAL PRESENTATIONS}

The OMMAT Study: Oncological Multisite Massage and Acupuncture Therapy to Combat the Side Effects of Routine Care

John R. Burns, Ana C. Perez Moreno, Jessica J. F. Kram, Jennifer T. Fink, John Scott Maul, Nancy Conway

Department of Integrative Medicine, Aurora Health Care; Aurora Research Institute; Aurora UW Medical Group; Center for Urban Population Health; University of WisconsinMilwaukee; Department of Oncology, Aurora Health Care

Background: Patients undergoing treatment for cancer face side effects (ie, pain, stress, neuropathy, and nausea) related to diagnosis, treatment, and distress of a life-threatening illness. Side effects are common, continue throughout treatment, can be serious, and impact quality of life and survival. Acupuncture and massage therapy have been shown to improve these side effects. Purpose: Explore whether massage and acupuncture therapy could reduce cancer treatment side effects across multiple sites.

Methods: Evidence-based clinical competencies were developed and applied to ensure quality of therapies provided across multiple Aurora Cancer Care clinics. Interventions were created for explicit types of cancer, patient conditions, and complaints. Pre/postintervention pain, stress, nausea, and neuropathy scores were recorded (scale: 0 [least] to 10 [worst]). Pre/postintervention scores were compared using paired t-tests.

Results: A total of 4367 acupuncture treatments (AT) and 4197 massage therapy (MT) treatments were provided to oncology patients in 2018. Comparing symptoms before and after AT revealed mean scores for pain (2.99 pre vs 1.42 post), neuropathy (2.75 vs 1.66$)$, stress ( 3.02 vs 1.18$)$, and nausea ( 0.45 vs 0.31 ) all were significantly different $(\mathrm{P}<0.001)$. F or the $\mathrm{m}$ ajority of AT there was a reported decrease in pain, stress, and neuropathy $(61.7 \%, 68.8 \%$, and $47.9 \%$, respectively). Analysis of pre-MT and post-MT symptom severity revealed mean scores for pain (1.61 pre vs 0.84 post), neuropathy ( 1.17 vs 0.77$)$, nausea $(0.26$ vs 0.16$)$, and stress (1.94 vs 0.93 ) all were significantly different $(\mathrm{P}<0.001)$. The impact of MT was greater on reducing stress $(42.5 \%)$ and pain $(34.4 \%)$.

Conclusion: Both therapies were effective at reducing symptom severity. AT was effective at decreasing both pain and stress, closely followed by neuropathy. MT impacted stress more than any of the other symptoms. Our findings s upport a larger randomized controlled trial to further assess the impact of massage and acupuncture therapy to combat the side effects associated with routine care across multiple cancer clinics.
Requirement for Electrical Cardioversion After Dofetilide Loading in Patients With Persistent Atrial Fibrillation Is Associated With Increased Recurrence

Bilal M. Omery, Khawaja Afzal Ammar, Arshad Jahangir, Andy Kieu, Tarek Ajam, Akshar Jaglan, Jasbir Sra, Atul Bhatia

Aurora Cardiovascular Services; Department of Internal Medicine, Aurora Health Care

Background: Atrial fibrillation (AF) recurrence after cardioversion following dofetilide loading with or without electrical cardioversion (EC) is reportedly $36 \%$ to $53 \%$. Although factors associated with $\mathrm{AF}$ recurrence are not well defined, it has been suggested that cardioversion during dofetilide loading without EC is associated with less AF recurrence.

Purpose: Investigate factors associated with AF recurrence within 1 year after cardioversion from persistent atrial fibrillation to sinus rhythm during dofetilide loading with or without EC.

Methods: We identified patients with persistent AF who had dofetilide loading from January 2014 to October 2017 and divided them into those who pharmacologically converted to sinus rhythm or required EC. Patients were longitudinally followed for 1 year for AF recurrence, stroke, torsades de pointes (TDP), and mortality.

Results: Out of 132 patients (mean age: $64.7 \pm 10.2$ years; $67 \%$ male; $20 \%$ with left ventricular ejection fraction [LVEF] of $35 \%$ or less; $80 \%$ with $\mathrm{CHA}_{2} \mathrm{DS}_{2}$-VASc score of at least 2), 65 patients $(49 \%)$ required EC. AF recurrence within 1 year occurred in 59 patients $(45 \%)$. One patient $(0.75 \%)$ experienced torsades de pointes the night after EC and subsequently died (mortality: $0.75 \%$ ) with heart failure. AF recurrence was more prevalent among patients who underwent EC (odds ratio [OR]: 2.09, 95\% CI: $1.04-4.20 ; \mathrm{P}=0.04$ ). AF recurrence in those who underwent EC continued to be higher in multivariate analyses, even after adjustment for $\mathrm{CHA}_{2} \mathrm{DS}_{2}$-VASc score, LVEF of $35 \%$ or less, and left atrial volume index greater than $48 \mathrm{~mL} / \mathrm{m}^{2}$. Stratum-specific odds ratios revealed this association progressively decreased in those with sleep apnea (OR: 6.5; $\mathrm{P}=0.01$ ), total daily dofetilide dose of 1000 micrograms (OR: 3.84, $\mathrm{P}=0.01$ ), left atrial volume index greater than $34 \mathrm{~mL} / \mathrm{m}^{2}$ (OR: $\left.3.15 ; \mathrm{P}=0.01\right), \mathrm{CHA}_{2} \mathrm{DS}_{2}$ VASc score of at least 2 (OR: 2.65; $\mathrm{P}=0.01)$, LVEF greater than 35\% (OR: 2.41; $\mathrm{P}=0.03$ ), male sex (OR: 2.48; $\mathrm{P}=0.04$ ), and no prior AF ablation (OR: 2.37; $\mathrm{P}=0.02$ ).

Conclusion: The need for EC after dofetilide loading was associated with increased AF recurrence incidence within 1 year despite adjustment for advanced substrate. The stronger association in certain clinical subsets warrants further investigation. 


\section{Can Magnetic Resonance Imaging Predict Pathologic Findings in Endometrioid Endometrial Cancer Patients?}

Elizabeth Dickson Michelson, Jessica J. F. Kram, Kayla Heslin, David Baugh, Vikram Bamra, Salik Nazeer, Jia Hao $\mathrm{Hu}$, Abhishek Shukla, Scott A. Kamelle

Department of Gynecologic Oncology, Aurora West Allis Medical Center; Aurora UW Medical Group; Center for Urban Population; Aurora Research Institute; Departments of Radiology, Pathology, and Gynecologic Oncology, Aurora St. Luke's Medical Center

Background: Endometrial cancer is the most common gynecologic cancer in the United States. Currently, hysterectomy and lymphadenectomy (more recently with sentinel lymph node removal only) are recommended for endometrioid endometrial cancer (EEC) patients at greater than low risk of cancer spread, with hysterectomy only recommended for patients at low risk. Our previously published risk schema, as well as risk schemas from other institutions, used tumor diameter (TD) and myometrial invasion (MI) to predict low-risk patients. Use of magnetic resonance imaging (MRI) may allow for TD and MI measurement preoperatively.

Purpose: Our study aimed to assess the feasibility of accurately measuring TD and MI in patients with endometrioid endometrial cancer using preoperative contrast-enhanced MRI.

Methods: We conducted a prospective pilot study on adult patients with confirmed diagnosis of complex hyperplasia with atypia or endometrioid endometrial cancer during August 2017July 2018. Further inclusion criteria required that patients must be English-speaking, be undergoing hysterectomy with one of two gynecologic oncologists, be eligible for MRI scan, be comfortable undergoing MRI scan with or without oral sedation, have less than severely compromised kidney function, and be able to undergo MRI at one medical center 7 days prior to hysterectomy. Image quality, as well as TD and MI image confidence, were measured on a scale of 1 to 3 (poor, moderate, excellent) by 3 radiologists. Basic descriptive statistics were performed.

Results: A total of 12 patients (mean age 58.8 years; mean body mass index: $35.6 \mathrm{~kg} / \mathrm{m}^{2}$ ) were enrolled and underwent preoperative MRI. Patient population was predominately white (91.7\%). On average, patients received their MRI within 33.3 days of diagnosis and surgery within 38.7 days. Using the pathology results for TD as the gold standard for comparison, at least 1 radiologist was able to predict largest TD within $5 \mathrm{~mm}$ for $41.7 \%$ of patients. All radiologists were not able to predict largest TD for any single patient. Similarly, using the pathology results for MI, at least 1 radiologist was able to predict MI within 5\% for $50 \%$ of patients. All radiologists were not able to predict MI for any single patient. Radiologists identified mean MRI image quality of 2.3. Overall, radiologists were relatively confident in TD and MI measurements, expressing 2.6 and 2.3 level of confidence, respectively.

Conclusion: Given the variation between radiologic evaluation, it is difficult to recommend preoperative MRI as a basis for measuring TD and MI.

\section{Epidemiologic Survey of Legionella Antigen Tests at} Aurora Health Care

Caroline P. Toberna, Hannah M. William, Dennis J. Baumgardner, Jessica J. F. Kram, Kayla Heslin

Department of Family Medicine, Aurora UW Medical Group; Center for Urban Population Health; Aurora Research Institute

Background: Legionella pneumophila is an aerobic, pathogenic, gram-negative bacterium that infects hosts through contaminated cooling towers and other sources of water. Human infection presents as potentially life-threatening pneumonia, otherwise known as Legionnaires' disease.

Purpose: Our study aimed to identify unrecognized outbreaks and geodemographic associations of Legionella in eastern Wisconsin.

Methods: We retrospectively reviewed relevant geodemographic information of patients who underwent Legionella urine antigen testing (LUAT) at a single eastern Wisconsin health system from January 2013 to December 2017. A random sample inclusive of all positive tests was reviewed. If a patient was ever identified as positive, only the encounter in which they had their first positive test was included in the analyses. For all other patients, only their first negative test was included. Negative cases were used to investigate significant geodemographic differences in patients testing positive versus negative. Data was further explored using chi-squared, 2-sample t-tests and stepwise regression, followed by binary logistic regression for multivariable analysis. Geographic information for positive cases was mapped to locate hotspots.

Results: Of all LUAT performed $(n=21,599), 0.68 \%$ were positive. Among those in the random sample $(n=11,652)$, positive LUAT cases were more prevalent in warmer months (June-November having $86.2 \%$ of all positive cases), younger, on average (59.4 years vs 67.7 years for negative cases), and disproportionately male $(70.3 \%$ vs $29.7 \%$ negative); $\mathrm{P}<0.0001$ for all. The prevalence of positive cases was higher among nonwhite race/ ethnicity $(1.91 \%$ vs $1.01 \% ; \mathrm{P}<0.0001)$. Nonwhite race/ethnicity did not remain significant on multivariable analysis, suggesting age \pm location may be an underlying predictor for infection. Overall, there were 13 zip codes in the city of Milwaukee containing 3 or more positive cases of Legionella. Within these zip codes, there were 58 positive cases. Of these, $36.2 \%$ were in zip codes of low socioeconomic status, $56.9 \%$ in zip codes of middle socioeconomic status, and the remaining $6.9 \%$ in zip codes of high socioeconomic status.

Conclusion: The prevalence of a positive LUAT in eastern Wisconsin was less than $1 \%$. Patients who are relatively younger, male, and nonwhite, and who are tested in warmer months, were more likely to test positive. This methodology, if done in real time, may complement public health detection of Legionnaires' disease outbreaks. Further study on built environments near clustered cases may reveal additional sources of infection. 


\section{Harmonizing Data From Multiple Institutions With a Common Data Model: An Oncologic Use Case for Risk Stratification}

Joseph M. Plasek, John A. Weissert, Kourosh Ravvaz

\section{Aurora Research Institute}

Background: The development and validation of clinical risk models increasingly relies on heterogeneous data from multiple institutions to increase the power and rigor of analysis. Harmonization across different sources requires adoption of the controlled vocabularies and standardized structure inherent in some common data models to achieve semantic interoperability. Both non-muscle invasive bladder cancer (NMIBC) therapy and research depend on accurate risk stratification, as NMIBC is a complex, diverse disease. Heterogeneous data, such as data from patients with NMIBC (eg, clinical, socioeconomic, genetic), is often collected from different sources that were generated for various purposes. The standardized structure of a common data model promotes the harmonization of multi-institutional data sets, which is necessary to validate existing risk models (eg, European Organization for Research and Treatment of Cancer [EORTC] NMIBC recurrence and progression models) and develop new high-performing NMIBC risk models. Bladder cancer is the ninth most common cancer, and NMIBC accounts for about $70 \%$ of new bladder cancer cases.

Purpose: To establish feasibility of a common data model for harmonizing multi-institutional NMIBC data and validating EORTC models.

Methods: In a retrospective cohort containing 509 and 1331 NMIBC patients from the University of Wisconsin-Madison and Aurora Health Care, respectively, we utilized the Observational Medical Outcomes Partnership (OMOP) common data model to transform the heterogeneous data sets into a standardized format in order to enforce consistent structure and semantics. EORTC risk factors for NMIBC include: number of tumors, tumor size, prior recurrence rate, tumor stage, carcinoma in situ, and grade. The EORTC risk scores were implemented using the patientlevel prediction tool from the OMOP community. Performance of EORTC risk scores was assessed using area under the receiver operator characteristic curve (AUC).

Results: The OMOP common data model was successfully implemented for NMIBC. Of 1840 patients, 861 (47\%) had a recurrence and 107 (6\%) progressed. AUC was 59.63\% (95\% CI: 56.93-62.33) for recurrence and 74.96\% (95\% CI: 69.35-80.57) for progression, consistent with prior studies.

Conclusion: The OMOP common data model provided a scalable infrastructure for harmonizing multi-institutional data that is useful for clinical risk stratification. There is demonstrable net benefit from implementing the OMOP common data model that supports advanced big data analytic techniques like machine learning to address existing clinical challenges.

\section{Zika Virus Selectively Kills Glioblastoma Progenitor Cancer Cells Through AXL Receptor}

Parvez Akhtar, Sam D. Zwernik, Amin B. Kassam, Richard A. Rovin

Aurora Research Institute; Aurora Neuroscience Innovation Institute
Background: An oncolytic virus must display tropism for the target cancer. This oncotropism is mediated by cell surface receptors that are differentially expressed by the cancer cell. Recent research into the mechanism of Zika virus (ZIKV)-induced microcephaly demonstrated that ZIKV has neurotropism for fetal neural progenitor cells, mediated by AXL, a member of the TAM (Tyro3, AXL, Mer) family of tyrosine kinase receptors. AXL is frequently overexpressed in majority of glioblastoma (GBM). We are attempting to repurpose ZIKV as an oncolytic virus that can specifically target and destroy glioblastoma through the AXL receptor.

Purpose: We hypothesized that ZIKV could preferentially target and kill glioblastoma progenitor cancer cells through the AXL receptor. Methods: ZIKV strain MR766 was propagated in Vero cells. Viral stock was titrated by plaque assays. Western blot was used to characterize AXL expression in 5 commercial glioblastoma cell lines and 10 patient-derived glioblastoma progenitor cell lines. Cell lines were infected with ZIKV and the percentage of infected cells quantified by flow cytometry using the pan-flavivirus anti-E protein Ab 4G2. Cells were treated with AXL inhibitor (R428) and/or human AXL antibody, then ablated for AXL using CRISPR knockout prior to ZIKV exposure. Infected cells were quantified by flow cytometry. Immunofluorescence microscopy was performed to visualize ZIKV entry and replication. Glioblastoma progenitor cells were terminally differentiated into different types of brain cells and tested for ZIKV infection.

Results: In this study, we found that AXL expression varies among our in-house patient-derived glioblastoma lines (7754, 7730,7714, 7753, 7978, 7759, 8034, 8035, 8049, and 7865) and commercial (ATCC) glioblastoma lines (LN229, A172, U251, and U87). Flow cytometry results confirmed that productive ZIKV infection only occurs in AXL-positive cells. We further found that ZIKV infection of our patient-derived glioblastoma cell lines was inhibited by AXL kinase inhibitor R428 in a dose-dependent manner. Furthermore, we found that blocking AXL receptor using AXL antibody significantly reduced ZIKV infection in glioblastoma in vitro. AXL knockout patient-derived glioblastoma lines $(7759,8034,8035,8049)$ completely blocked ZIKV infection and significantly inhibited viral replication. Our results highlight the dual role of AXL during ZIKV infection of glioblastoma: promoting viral entry and facilitating productive replication. We also found that ZIKV is unable to replicate in terminally differentiated glioblastoma progenitor cancer cells, suggesting selectivity for stem cells.

Conclusion: These results clearly suggest that ZIKV displays oncotropism for glioblastoma progenitor cancer cells, mediated primarily through $\mathrm{AXL}$ receptor.

\section{Weighting Risk Factors for Justice Involvement in Patients Served by a Large Health System}

Sarah E. Reimer, Noah C. Pearce, Christopher R. Beal, Andrew R. Marek, Maharaj Singh

Center for Urban Population Health; Aurora Research Institute

Background: Mass incarceration in the United States has caused incarceration to be an expected life stage in some communities. Wisconsin incarcerates black males at nearly twice the national average, and Milwaukee has the most heavily incarcerated zip code in the country. This study compares justice-involved and nonjustice-involved Milwaukee County residents served by a large health care system. 
Purpose: This study aims to characterize justice-involved and non-justice-involved populations.

Methods: Electronic health records from Aurora Health Care were used to identify a cohort of 564,988 patients, age 18-100 years by end of 2017, that lived in Milwaukee County for 360 or more days and had 2 or more in-person encounters between 2008 and 2017. Incarceration records were obtained from correctional facilities administered by Milwaukee County and the state of Wisconsin between May 2008 and June 2018. Unique persons were identified within these incarceration records $(n=166,563)$ and cross-referenced to Aurora's electronic health record to determine which people had ever been patients $(n=109,723)$. The cohort was separated into two groups: justice-involved $(n=55,118)$ and not justice-involved $(n=509,870)$, based on the presence of incarceration records for each cohort member. Next, clinical data from the electronic health record was incorporated into the cohort, and the two groups were compared using descriptive statistics.

Results: In all, $43.5 \%$ were male, $66 \%$ white, and $24 \%$ African American, with the remaining belonging to Hispanic and other races. Mean age of the total population was 48.6 years (SD: 19.5). Odds ratios (OR) identified major independent predictors of justice involvement to be male gender (OR:4.1 [4.0-4.2]), African American race (OR: 3.7 [3.5-3.8]), Medicaid insurance status (OR: 3.3 [3.2-3.4]), Medicare insurance status (OR: 1.1 [1.1-1.2]), tobacco use (OR: 2.5 [2.4-2.5]), and drug use (OR: 2.0 [2.0-2.1]). Patients who had a primary health care provider (OR: $0.7[0.7-$ $0.7]$ ), were between 50 and 70 years old (OR: 0.5 [0.5-0.5]), or were older than age 70 (OR: 0.1 [0.1-0.1]) were less likely to have been recently justice-involved. Each odds ratio noted herein had a P-value of $<0.01$.

Conclusion: Several demographic features associated with justice involvement in a health system population were identified. Future work aims to reduce potential confounders, such as patient neighborhood, and to delineate the impact of justice involvement on health metrics related to diseases known to be of higher prevalence in the justice-involved population.

\section{Cardiac Catheterization and Risk Factors of Mortality}

Reji Babygirija, Maharaj Singh, Richard A. Rovin, Kessarin Panichpisal

\section{Aurora Research Institute; Aurora Neuroscience Innovation Institute}

Background: Cardiac catheterization is the gold standard to diagnose and treat cardiovascular conditions, including pulmonary hypertension. Estimated annual rates for cardiac catheterization, for both sexes, range from 171 to 650 per 100,000 population ( $>20$ years old). The risks and complications following cardiac catheterization are related to the patient's concomitant conditions as well as medications. There are limited data about the factors that influence the risk of serious adverse events after cardiac catheterization.

Purpose: Identify the risk factors of mortality for patients undergoing cardiac catheterization procedure.

Methods: This is a single-center retrospective data collection study on patients who underwent cardiac catheterization between January 2008 and March 2017. Demographics, comorbidities, and medications were included in Cox proportional hazards model.

Results: A total of 11,062 patients were included in the study. Of these, $66 \%$ were male, $89.9 \%$ were white, $8.1 \%$ were black, and mean age of this cohort was $66.3 \pm 12.2$. Overall mortality rate was $19.4 \%$. Main predictors of mortality were: age (in 10year increments) at the time of cardiac catheterization procedure (hazard ratio [HR]: 1.71 [1.62-1.79]; $\mathrm{P}<0.001$ ); Hispanic ethnicity (HR: 0.65 [0.52-0.81]; $\mathrm{P}=0.002$ ); diabetes mellitus (HR: 1.74 [1.59-1.91]; $\mathrm{P}<0.001$ ); prior myocardial infraction (HR: 1.27 [1.16-1.40]; $\mathrm{P}<0.001)$; prior coronary artery disease (HR: 1.61 [1.27-2.05]; $\mathrm{P}=0.001$ ); hypertension (HR: 1.23 [1.061.42]; $\mathrm{P}=0.005$ ); smoking (HR: 1.35 [1.20-1.53]; $\mathrm{P}<0.001$ ); history of stroke (HR: 1.53 [1.38-1.70]; $\mathrm{P}<0.001$ ); aspirin (HR: 0.39 [0.33-0.45]; $\mathrm{P}<0.001$ ); antiplatelet (HR: 0.30 [0.25-0.36]; $\mathrm{P}<0.001$ ); angiotensin-converting enzyme (ACE) inhibitors (HR: 0.80 [0.73-0.89]; $\mathrm{P}<0.001)$; angiotensin II receptor blockers (ARB) (HR: 0.66 [0.58-0.76]; $\mathrm{P}<0.001$ ); statin (HR: 0.65 [0.57$0.74]$; $\mathrm{P}<0.001$ ); nonstatin medication (HR: 0.86 [0.78-0.96]; $\mathrm{P}=0.0088$ ); and beta blocker (HR: 0.81 [0.72-0.90]; $\mathrm{P}=0.0002$ ).

Conclusion: This study revealed that the mean survival for cardiac catheterization patients was more than 6 years. Risk factors for mortality after cardiac catheterization were age, ethnicity, diabetes, myocardial infraction, coronary artery disease, hypertension, smoking, and prior history of stroke. However, medications, including aspirin, antiplatelets, ACE inhibitors, ARB, statin, nonstatins, and beta blockers, were found to be protective.

\section{Evaluation of Beta Blockers and Renin-Angiotensin Inhibitors for the Reduction of Anthracycline-Induced Cardiotoxicity}

Evan Mueller, Sarah Zukkoor Zorn, Rachel Pedersen, Vinay Thohan

Department of Pharmacy, Aurora Health Care; Tendick Center for Advanced Heart Failure Therapies

Background: Anthracyclines are known to cause dose-related cardiotoxicity. Clinical trials suggest that beta blockers and renin-angiotensin inhibitors may attenuate anthracycline-induced cardiotoxicity. However, studies are small with confounding factors, and guidelines remain inconsistent regarding optimal use of these medications for cardioprotection in oncology.

Purpose: To evaluate the role of beta blockers and reninangiotensin inhibitors in reducing anthracycline-induced cardiotoxicity.

Methods: A retrospective review was conducted for patients, age 18 years or older, who received anthracyclines at Aurora Health Care from January 2012 to December 2017. Patients on beta blockers or renin-angiotensin inhibitors prior to chemotherapy were compared to those not on these medications. The primary outcome was incidence of reduced left ventricular ejection fraction (LVEF), defined as time to first LVEF less than $53 \%$ by echocardiogram. Exclusion criteria included baseline LVEF of less than $53 \%$ and no baseline or postanthracycline echocardiogram. Continuous variables are expressed as means and standard deviations and compared between groups via Student's t-test. Categorical variables are shown as frequency and compared between groups via chi-squared test. Competing risks analysis was used to estimate rates of reduced LVEF, and proportional subdistribution hazards regression was used to identify a final multivariable model.

Results: A total of 799 patients were included for analysis. Patients on beta blockers or renin-angiotensin inhibitors at anthracycline start were older, had higher body mass index, 
lower cumulative life dose, and lower alcohol use. Additionally, they had higher rates of atrial fibrillation/flutter, coronary artery disease, diabetes, heart failure, hyperlipidemia, and hypertension. A final multivariable model identified age of $\geq 65$ years (hazard ratio [HR]: 2.11, 95\% CI: 1.27-3.50; $\mathrm{P}<0.01$ ), increasing cumulative life dose (HR per 50-unit increase: 1.13, 95\% CI: 1.02-1.24; $\mathrm{P}<0.02$ ), male sex (HR: 2.36 , 95\% CI: $1.42-$ 3.93; $\mathrm{P}<0.01$ ), and prechemotherapy LVEF of $<60 \%$ (HR: 3.48 , 95\% CI: $2.13-5.69 ; \mathrm{P}<0.01)$ as independent predictors of nonnormal LVEF.

Conclusion: Within this cohort, patients on beta blockers or renin-angiotensin inhibitors at start of anthracycline therapy did not have a significant difference in incidence of postchemotherapy LVEF of $<53 \%$ compared to patients not on those medications. Of note, the patients on medications had a higher incidence of preexisting cardiac disease, which may mask the potential cardioprotective benefits, confounding the results.

\section{POSTER PRESENTATIONS}

Real-Life Impact of Physician Awareness of a Positive Stool DNA Test on Subsequent Colonoscopy Outcome in Colorectal Cancer Screening

\section{Julien Fahed, Aboud Affi}

\section{Department of Gastroenterology, Aurora Health Care}

Background: A recent study of 244 subjects looked into clinical outcome of colonoscopy based on the physician's awareness of the positive stool DNA test result. The study compared unblinded unmonitored colonoscopy with blinded monitored colonoscopy.

Purpose: We present a larger real-life study on the impact of physician awareness of a positive stool DNA test on colonoscopy results.

Methods: Chart review of 1413 patients who had a positive stool DNA followed by documented subsequent colonoscopy was done. Attention was paid to the indication of the procedure in the colonoscopy report. A total of 5 patients were excluded; 3 of these had unknown indication and 2 a finding of "other carcinoma." Findings were recorded and patients were divided into two groups. Group 1 included patients who had indication of "stool DNA testing" or its equivalent, while group 2 included all the other indications. Only the index lesion was recorded. Advanced adenoma was defined as an adenoma larger than 10 $\mathrm{mm}$ or that had high-risk features such as villous transformation or high-grade dysplasia. Nonadvanced adenoma was defined as an adenoma smaller than $10 \mathrm{~mm}$ with no high-risk features. Statistical analysis with chi-squared was done to compare the proportions. A P-value less than 0.05 was considered statistically significant.

Results: Of the 1408 patients, 979 (70\%) had an indication of "stool DNA testing" or its equivalent, while 429 (30\%) had another indication (to which we assumed the provider was not aware of the positive stool DNA testing). Results of group 1 and group 2 were as follows: Adenocarcinoma was found in $1.8 \%$ vs $1.4 \%$, respectively $(\mathrm{P}=0.56)$; advanced adenoma was found in $24.8 \%$ vs $21.2 \%$, respectively $(\mathrm{P}=0.14) ;>10-\mathrm{mm}$ adenoma was found in $7.25 \%$ vs $6.99 \%$, respectively $(\mathrm{P}=0.86)$; and nonadvanced adenoma was found in $28.7 \%$ vs $31.2 \%$, respectively $(\mathrm{P}=0.57)$. Finally, no adenomas were found in $37.4 \%$ vs $39.2 \%$, respectively $(\mathrm{P}=0.33)$.
Conclusion: Following a positive stool DNA test, 1 out of 3 colonoscopies are done without the physician's awareness of the positive result. Fortunately, there was no difference in the outcome of colonoscopy whether the physician was aware of the positive result or not.

\section{Calling Urgent and Emergent Cesarean Sections: Are We on Time?}

Naomi Light, Jessica J. F. Kram, Kayla Heslin, Cheryl Bauer, Mariah Peterson, Carla Kelly

Departments of Obstetrics and Gynecology, Women's Health Services, and Labor and Delivery, Aurora Sinai Medical Center; Aurora UW Medical Group; Center for Urban Population Health; Aurora Research Institute

Background: In October 2016, our health care facility adopted a new classification system for cesarean sections in an effort to have on-time cesarean deliveries among typically unscheduled patients with time-sensitive cesarean sections. Patients with timesensitive cesarean sections include those with an immediate threat to life (emergent) or those with maternal or fetal compromise that is not immediately life threatening (urgent). Even when a cesarean delivery is deemed emergent or urgent (ie, timesensitive), patient-, staff-, anesthesia-, and procedure-related delays may occur.

Purpose: Our quality improvement study aimed to assess whether caregiver education would improve on-time cesarean deliveries.

Methods: We retrospectively studied all emergent ( $<15$ minutes) and urgent ( $<30$ minutes) cesarean sections within one labor and delivery unit. Patients 6 months prior to caregiver education were compared with those following nursing and provider education. Nursing and provider education (September 2017-October 2017) focused on development of communication pathways and reorientation to decision-making timelines. To compare groups, descriptive statistics and multivariable linear regression were used to predict time to incision.

Results: A total of 149 patients pre-caregiver education and 185 patients post-caregiver education were included. Patients in the two groups did not differ by age, race/ethnicity, or body mass index (BMI). On univariate analysis, mean time from decision to incision, as well as mean time from decision to anesthesia in patients who did not already have anesthesia initiated, significantly improved $(\mathrm{P}<0.01)$. Additionally, delays significantly improved $(67.1 \%$ vs $53.5 \% ; \mathrm{P}=0.01)$, although documented reasons for delays did not $(\mathrm{P}=0.14)$. Overall, only $21.1 \%$ of delayed patients $(n=199)$ were emergent cesarean sections. Multivariable regression suggested case classification, patient delay, BMI, and time to anesthesia, but not the educational intervention, significantly predicted time to incision $(\mathrm{P} \leq 0.01$; $\mathrm{R}^{2}=0.86$ ).

Conclusion: Although an improvement in decision-toincision time was observed, it was unrelated to the educational intervention performed. Even so, concurrent nursing education initiatives aimed at improving cesarean section timing may have contributed to on-time emergent and urgent cesarean deliveries. Ultimately, ongoing education and team collaboration should continue in order to further improve cesarean delivery timing and patient care. 


\section{Criteria for Conversion From a Basal-Bolus Insulin Regimen in Patients With Type 2 Diabetes to a Regimen of a Glucagon-Like Peptide-1 Receptor Agonist With Basal Insulin}

\section{Christopher Klink, Kelsey Gardetto, Thomas Dilworth}

\section{Department of Pharmacy, Aurora Health Care}

Background: Diabetes mellitus is a metabolic disease in which the body's production and response to insulin is altered. Type 2 diabetes mellitus is a subset of the disease in which the pancreas continues to produce insulin; however, the level of endogenous insulin production decreases over time. Many patients with type 2 diabetes mellitus eventually require basal-bolus insulin as part of their medication regimen; however, this regimen is complex and increases the risk of hypoglycemia and weight gain. A newer regimen for type 2 diabetes mellitus includes the combination of a glucagon-like peptide-1 receptor agonist (GLP-1RA) plus basal insulin. This regimen is less complex, safer, promotes weight loss, and has the potential to reduce cardiovascular risk. This regimen is incorporated in the American Diabetes Association guidelines as part of the step therapy for diabetes. What is less well known is which patients, if any, may be successfully de-escalated from basal-bolus insulin to this regimen.

Purpose: To identify patient characteristics that predict a successful conversion from a basal-bolus insulin regimen to a basal insulin plus GLP-1RA regimen.

Methods: A retrospective case series was performed on 32 patients with type 2 diabetes mellitus. Of the 32,24 patients successfully made the conversion from a basal-bolus insulin regimen to GLP1RA plus basal insulin; 8 patients were unsuccessful. Unsuccessful conversions were defined as discontinuation of the GLP-1RA due to intolerance, expense, or clinical decline seen in lab test values. Patient characteristics, including weight, hemoglobin Alc, and blood glucose (average and range), were tracked. C-peptide level, years since diagnosis, and insulin requirements also were analyzed to identify if there may be a correlation with patients who could successfully be converted.

Results: Patients who successfully converted to a GLP-1RA plus basal insulin regimen had an average weight loss of $5.5 \pm 2.0 \mathrm{~kg}$ and a reduction in average blood sugar of $16.3 \pm 51.2 \mathrm{mg} / \mathrm{dL}$ at 3 months. They also had an average reduction in A1c of $0.83 \%$; in comparison, patients who were unsuccessful had an average increase in Alc of $0.625 \%$. The average C-peptide value for successfully converted patients was $2.77 \mathrm{ng} / \mathrm{ml}$ compared to $0.97 \mathrm{ng} / \mathrm{ml}$ for patients who failed the new regimen due to clinical decline.

Conclusion: Patients with type 2 diabetes mellitus presently managed on basal-bolus insulin can be successfully converted to a GLP-1RA plus basal insulin if appropriately screened and selected.

\section{Vagal Response During Pulmonary Vein Isolation With Cryoballoon Ablation}

Janet C. Tsang, Lynn Erickson, Imran Niazi, Jasbir S. Sra, M. Eyman Mortada

\section{Aurora Cardiovascular Services; Aurora Research Institute}

Background: Vagal nerve response, seen during cryoballoon ablation of the pulmonary veins, can be attributed to the ablation's effect on the ganglionated plexi. This effect is demonstrated with drops in blood pressure and heart rate during pulmonary vein isolation with cryoballoon ablation.

Purpose: To evaluate surrogate markers for vagal response during pulmonary vein isolation with cryoballoon ablation.

Methods: A total of 22 patients with paroxysmal atrial fibrillation underwent pulmonary vein isolation with a $28-\mathrm{mm}$ second-generation cryoballoon using a 3-minute freeze technique. Two or more lesions were performed for each vein. Initial blood pressure, nadir blood pressure, time to reach -30 degrees, time to reach -40 degrees, time from start of thaw to reach zero degrees (TT0), time from start of thaw to the nadir blood pressure (TTNBP), total freeze time, minimal temperature reached, and time from start of freeze to vein isolation by electrogram were recorded. Clustered data for each vein were analyzed using the common slope and linear intercept mixed model. Results: All patients (mean age: $62.5 \pm 8.4$ years; male sex: $69 \%$, body mass index: $31.7 \pm 5.7$; mean $\mathrm{CHA}_{2} \mathrm{DS}_{2}$-VASc score: $1.8 \pm$ 1.2 ; white race: $100 \%$; hypertension: $56 \%$; coronary artery disease: $38 \%$; sleep apnea: $50 \%$; mean left atrial volume index: $58.6 \pm$ $17.8 \mathrm{ml} / \mathrm{m}^{2}$; left ventricular ejection fraction of $\geq 55: 100 \%$ ) had paroxysmal atrial fibrillation. Univariate analysis demonstrated that the best surrogate marker for vagal response was the TT0 in the inferior veins. The drop in blood pressure increased up to fourfold, with a onefold increase in thawing duration (TT0 in left inferior vein: 3.040, $\mathrm{P}<0.020$; TT0 in right inferior vein: 4.261, $\mathrm{P}<0.046$ ). Average TT0 and drop of systolic blood pressure were 7.5 seconds and $21 \mathrm{mmHg}$, respectively, in the left inferior vein, and 7 seconds and $20.8 \mathrm{mmHg}$, respectively, in the right inferior vein. The second marker was the TTNBP, seen only in the right superior vein, with average time of 86.6 seconds, causing an average of systolic blood pressure drop of $24.6 \mathrm{mmHg}$. A threefold increase in TTNBP caused onefold increase in blood pressure drop $(0.323, \mathrm{P}<0.037)$.

Conclusion: Vagal response during cryoballoon ablation was seen mainly during isolation of the inferior pulmonary veins.

\section{Evaluation of Opioid Prescribing Habits and Morphine Milligram Equivalent Trends at Aurora Health Care}

Brent Davis, Prati Wojtal, Joel Pietryga, Barry McClain, Tristan F. Edwards

\section{Department of Pharmacy, Aurora Health Care}

Background: Aurora Health Care's opioid stewardship committee (OSC) was created to optimize and standardize pain management in patients while minimizing the use of opioids and reducing the number of patients who develop opioid use disorder. In 2016, the Centers for Disease Control and Prevention (CDC) established guidelines for prescribing opioids, but execution and awareness of the current guidelines is inconsistent across the system. An initiative of the OSC is to ensure that all providers are following the CDC opioid-prescribing guidelines. The specific goals of this committee are to: 1) reduce the total amount of opioids prescribed in morphine milligram equivalents (MMEs); 2) reduce the number of patients co-prescribed opioids and benzodiazepines; and 3) minimize the number of patients started on chronic opioid agonist therapy.

Purpose: An opioid-prescribing dashboard was designed to assess baseline opioid-prescribing practices and the goals of the OSC. The opioid-prescribing dashboard provides leadership visibility to MME prescribing trends and prescribing practices deviating outside the CDC opioid-prescribing guidelines.

Methods: OSC members identified important definitions and metrics for reporting in the dashboard. Metrics were adopted from literature 
reviews of successful opioid dashboards at other health systems and in harmony with $\mathrm{CDC}$ opioid-prescribing guidelines. MME reports are the key catalyst to the dashboard's analysis. In 2018, Aurora linked daily MME calculations to opioid prescriptions in its electronic health record (EHR). In October 2018, CDC MME conversion factors were used to validate EHR's daily MME calculations for opioids prescribed. Incorrect or missing MME calculations were identified and addressed. An initial report was developed allowing for baseline reporting during November 1, 2018January 31,2019 . The data was filtered by provider, specialty, and service area and then manipulated for reporting on the dashboard.

Results: During the 3-month period, 105,874 opioid prescriptions, with an average daily MME of 40, were prescribed. The average monthly MME total was 21.8 million, with $29 \%$ and $15 \%$ of patients tallying a daily MME of $\geq 50$ and $\geq 90$ on opioids. Aurora averaged 1229 new chronic opioid patients each month and currently accounts for 8307 total chronic opioid patients. In January 2019, $68 \%$ of patients prescribed opioids were prescribed benzodiazepines between September 1, 2018, and January 31, 2019.

Conclusion: The dashboard identifies trends and areas for improvement in prescribing opioids with the intention to encourage safe and appropriate prescribing for Aurora patients.

\section{Strengthening the Culture of Well-Being in Graduate Medical Education}

Jacob Bidwell, Tricia La Fratta, Deborah Simpson, Nicole Eull

Departments of Family Medicine, Graduate Medical Education, and Academic Affairs, Aurora Health Care

Background: Physician burnout is a safety, quality, and workforce issue. As a result, well-being is an Accreditation Council on Graduate Medical Education's (ACGME) accreditation requirement for the sponsoring institution and individual programs. Aurora Health Care's graduate medical education (GME) leaders convened a retreat of key stakeholders (program directors, resident council leaders, system leaders) to develop a well-being strategic plan. Each program completed the ACGME's Inventory of Elements of Your Program's Well-Being plan, and GME leaders completed Inventory of Elements of Your Institution's Well-Being plan. At the retreat cross-cutting findings were identified, with action items identified.

Purpose: To improve our resident/fellow well-being scores through the development of clear GME protocols, procedures, and initiatives with pre/post metrics.

Methods: Major GME strategic action items focused on aligning physician contracts with teaching roles, procuring funds for a medical education well-being position, developing direct access for trainees to behavioral health, developing a systemwide budget for ensuring a standard for adequate time and space for pursuing wellbeing, and developing a crisis communication protocol. The impact of our interventions was measured by the evidence-based Mayo Well-Being Index (MWBI) and the ACGME's well-being survey.

Results: All action items have been implemented, including appointment of a 0.80 FTE well-being director. Our resident/fellow wellness scores were positive. MWBI results showed a $4.6 \%$ decrease $(17.3 \%$ to $12.7 \%)$ of residents/fellows $(n=158)$ reporting high levels of distress over the last 11 months, well below the national average of $34.9 \%$ (>1700 residents/fellows). Our ACGME well-being survey results were within normal range per national metrics.
Conclusion: All GME action items were completed, with the percentage of at-risk scores decreasing by $4.6 \%$ over an 11 -month period. Going forward, GME will continue to monitor MWBI and ACGME scores and support residency/program-specific interventions as part of a continuous improvement.

\section{Understanding the Top Health Issues Facing Milwaukee County}

David Frazer, Carrie Stehman, Maddie Johnson, Kayla Heslin, Elizabeth A. Duthie, Marianne Klumph

Center for Urban Population Health; University of Wisconsin School of Medicine and Public Health; Aurora Research Institute; Aurora UW Medical Group; University of WisconsinMilwaukee

Background: Health is determined by complex interactions among socioeconomic factors, health behavior, health care, and physical environment. Since 2012, the Milwaukee Health Care Partnership's triennial Community Health Needs Assessment (CHNA) has reported the top health issues identified by residents and community leaders in Milwaukee County. The CHNA serves as the foundation from which hospitals, community health centers, and local health departments develop their community health improvement strategies.

Purpose: To better understand the health of Milwaukee County and what influences it.

Methods: The CHNA is informed by three key sources: Community Health Survey (a 14-minute, phone-based survey of Milwaukee County residents that addresses adult/child health risks, health behaviors, and community health needs); key informant interviews (conducted with health experts and community leaders to identify community health needs, contributing social factors, and organizations best suited to address those issues); and Health Compass Milwaukee (a compilation of publicly available data, including sources mentioned above, on a recently launched website). We utilized all three sources to create the CHNA to help understand the burdens, disparities, inequities, and determinants that contribute to health issues.

Results: The top 5 issues of greatest concern were chronic disease, mental health, substance use, violence, and access to health care. Among chronic disease conditions, heart disease was identified as the leading cause of death in Milwaukee County, with a premature death rate of 1228 per 100,000 lives, which is higher than the rest of Wisconsin. Among mental health outcomes, suicide rates in Wisconsin jumped 55.6\% from 2000 to 2017 but remained relatively level in Milwaukee County. Regarding substance use in Milwaukee County, the opioid-related overdose death counts are concerning, with an increase from 144 in 2012 to 337 in 2017. In terms of violence against individuals that inflicts physical harm, Milwaukee County (945.1 violent crimes per 100,000 individuals) has a higher rate of violence than the rest of Wisconsin (282.9). On a positive note, regarding health care access, the Affordable Care Act spurred Milwaukee County's uninsured rate to drop from $12.6 \%$ in 2013 to $7.2 \%$ in 2017.

Conclusion: Health is complex. It is our hope that this assessment and website will help amplify providers and community conversations about the importance of cross-sector collaboration for health improvement. 


\section{Impact of Obstetrics and Gynecology Resident Workload and Protected Time Interventions on Well Being}

\section{Morgan S. Altinok, Naomi Light, Deborah Simpson, Carla Kelly}

Department of Obstetrics and Gynecology, Aurora Sinai Medical Center; Department of Family Medicine and Academic Affairs, Aurora Health Care; Aurora UW Medical Group

Background: Between $22 \%$ and $60 \%$ of practicing physicians are reported to have experienced burnout, with $\mathrm{OB} / \mathrm{GYN}$ resident burnout having been reported at $90 \%$. Duty-hour limitations were implemented for patient safety and have been associated with some increase in overall resident quality of life and potential sacrifices in resident education and patient care. Contributors to burnout (and drivers of engagement) include workload and job demands, lack of control and flexibility over personal schedule and workload, poor work-life integration, and numerous check box requirements (filling out surveys, module requirements, duplicates, paperwork). Purpose: To improve well-being and decrease burnout among OB/ GYN residents by implementing workload changes and protected time for wellness.

Methods: Effective July 2017, we changed 3 resident workload protocols. 1) For weekend rounding, residents continue to round on all antepartum and gynecology patients at the end of each 24-hour shift, but faculty became responsible for completing all postpartum rounding. 2) Weekday postpartum rounding was redistributed, and the number of patients decreased from $>10$ to maximum of 6-7 patients per junior resident. 3) No resident service obligations on Sundays with 2 months with no residents on night float. Quarterly wellness mornings began in September 2017, using protected education time for residents. We measured pre/post changes in well-being through a brief well-being check in card (WBCIC) and Mayo Well-Being Index (MWBI).

Results: Resident response rates ranged from 10 to 12 . WBCIC baseline (September 2017), midintervention (May 2018), and postintervention (December 2018) scores on item "Time spent on wellbeing" improved from 1.6 to 2.1 to 2.3 (scale: 1=pitiful to $4=$ excellent). The item "The work I do is meaningful to me" began at 4.9, then remained constant at 5.2 (scale: 1=strongly disagree to $7=$ strongly agree). The MWBI identifies those at risk of adverse outcomes due to poor quality of life, burnout, and suicidal ideation if their score is $>5$. MWBI scores remained more than 1.2 points below the 5.0 cutoff, ranging from 1.2 to 1.8 during report period with seasonal variation (residents were less well in winter). Wellness mornings were most frequently used for sleep, connecting with family/ friends/other residents, exercise, and personal health appointment.

Conclusion: The workload interventions were associated with a positive trend in overall well-being and finding meaning in work, making progress towards our goal of creating a workplace dynamic where burnout is combated and well-being is promoted.

\section{Opportunity for Protected Administrative Time Improved Resident Well-Being and Patients' Experience of Care}

Vy T. Dinh, Thomas J. Harrington, Joseph Vogelgesang, Abdulrehman Siddiqui, Catherine de Grandville, Will Lehmann, Deborah Simpson

Department of Family Medicine, Aurora Health Care; Aurora UW Medical Group; Department of Academic Affairs, Aurora Health Care
Background: Well-being is a critical issue for health care, with burnout rates measured at up to $63 \%$ among family physicians. A recent meta-analysis of burnout and patient experience studies found a strong association between resident and physician wellness and quality of patient care. Nationally, indirect patient care responsibilities (visit notes, inboxes, phone calls) has been identified as a significant contributing factor for burnout. Primary care physicians who spend, on average, 6 hours/week on electronic health record work outside normal clinical time are 3 times more likely to report burnout. Our family medicine residents identified lack of time to manage patientrelated "in-boxes" as a barrier to their well-being.

Purpose: To determine if allocating one-half day/week to complete indirect patient care tasks reduced residents' perceived administrative tasks burden and improved the patient's experience.

Methods: Once clinical schedules were established, residency program leaders (faculty, clinic, chief residents) allowed residents on noncall rotation blocks to notify their preceptor if they wanted to take a half-day to perform administrative tasks. Ongoing and repeated communication using multiple forums and formats was provided to clarify the purpose and process for using this allocated time. The effectiveness of this intervention was evaluated using two outcomes (CG-CAHPS, Mayo Well-Being Index) and two process surveys (end-of-rotation, resident wellness) as pre/post metrics.

Results: On process survey results, the percentage of residents reporting that "things are piling up so high I don't feel I can overcome them" decreased by $16 \%$ from $>1$ times/week to $1-2 /$ month. Further analysis revealed that as the number of half-days taken increased (from 4.0 to 4.5 half-days/rotation), residents felt more under control ( 1.4 to 1.7 , with $1=$ strongly agree to $5=$ strongly disagree). Outcome measures demonstrated a positive pre/post change: Mayo Well-Being Index scores showed 1.2 positive change (on 6-point scale); CG-CAHPS percentiles in our two family medicine residency clinics increased (between-visit communication by 4 and 5 points, respectively, and test-results communication by 5 and 7 points, respectively).

Conclusion: Our results demonstrate that protected time for administrative tasks improves residents' sense of control and wellbeing. Patient quality care scores also improved, highlighting the strong association between physician wellness and patient's experience of care.

\section{Running and Rapping Our Way to Wellness: Internal Medicine Residency Approach to Preventing Burnout}

Siri Neelati, Prakash Nallani, Kathy Scigacz, Richard Battiola, Tanya Shah, Xiaoxiao Qian, Deborah Simpson

Department of Internal Medicine, Aurora Sinai Medical Center; Aurora UW Medical Group; Department of Family Medicine and Academic Affairs, Aurora Health Care

Background: Physician burnout has led to increased cardiovascular disease, shorter life expectancy, problematic alcohol use, depression, and suicide. A recent meta-analysis on burnout and patient experience highlighted the strong association between physician wellness and the quality of patient care. Unfortunately, burnout in internal medicine ranks among the highest of all specialties, with rates up to $76 \%$.

Purpose: To facilitate resident mental and physical well-being through initiatives focused on supportive colleague relationships and exercise.

Methods: We implemented two related interventions. 1) RAPS (Resident Advisor for Peer Success) is a structured peer advisor 
program for incoming interns to jump-start a "connection" with their continuity clinic team shortly after match day. This early and sustained contact provides interns with an immediate resource for questions/support and ongoing connections with senior residents and a faculty advisor. 2) Fit4Life intervention continued to build/ support colleague relationships through personal fitness. Wellness challenges between trainee years were held to promote physical health per American Heart Association (AHA) exercise guidelines. Three measures - a Fit4Life survey, semi-structured interviews with interns, and Mayo Well-Being Index (MWBI) - were used to evaluate process and outcomes.

Results: Fit4Life pre-, during, and postchallenge data showed that less than $20 \%$ of internal medicine residents met AHA guidelines: 150 minutes/week of aerobic physical activity. Challenge period results showed a modest but not significant increase in exercise. Average completion rate was $83 \%$ of internal medicine residents. Exercise, per AHA recommendations, was paired $>50 \%$ of time with another activity (eg, watch/listen to television, movies, music, podcasts; connecting with family/friends). RAP intern interviews (13 of $13[100 \%]$ ) revealed that $85 \%$ were supportive of RAPS and $100 \%$ met weekly with RAPS team member(s) at continuity clinic. MWBI scores throughout intervention were well below instruments established at risk level for burnout (5.0), with below burnout scores ranging from 3.1 preintervention (February 2018) to 2.3 postintervention (December 2018).

Conclusion: The results of our well-being interventions focused on supportive colleague relationships through RAPS and exercise reveal high participation rates and support but no significant impact on physical or mental health as measured by Fit4Life and MWBI.

\section{Health Care Utilization as a Proxy to Introduce a Novel Disease Screen in Pediatric Populations}

\section{Angela A. Navarrete-Opazo, Maharaj Singh, Mindy R. Waite, Sheldon R. Garrison}

\section{Aurora Research Institute}

Background: The global economic burden of treating genetically distinct conditions is growing rapidly. An interest in prophylactic treatment and pharmacogenetic decision-making has emerged in concert with an increasing usage of genetic tools, such as prenatal genetic testing, biomarker assays, and personal genomic tests, to presymptomatically identify these conditions. However, little is known about the optimal timing of these approaches to best impact patient care and, where applicable, to consider the cost of care in providing prophylactic treatment.

Purpose: To determine the optimal developmental timepoint in early childhood to screen for certain health conditions presymptomatically and measure the financial impact of the resulting clinical actions.

Methods: A retrospective review of vaccination compliance, as a proxy for health care utilization, of the 3,969,953 eligible individuals from 2012 through 2018 using de-identified data from the Wisconsin Immunization Registry. Further analysis across racial groups was assessed, and the financial impact of changes in cost of care. Concurrently, a parental survey was conducted to determine preference for the timing and disease inclusions of genetic tests.

Results: Overall vaccination rates revealed maximal compliance during the first 6 months of visits, with the highest compliance rate at month $4(71.2 \%)$. However, this rate significantly differed when racial groups were assessed independently, with compliance in Caucasian children highest at 4 months $(80.0 \%)$ and all other racial backgrounds highest at 2 months $(66.3 \%-72.6 \%)$.

Conclusion: Results demonstrated that health care utilization is greatest within the first 4 months after birth, suggesting an optimal time to administer tests designed to detect health conditions presymptomatically.

\section{Incidence of Atypia or Malignancy on Surgical Excisional Biopsy From Benign Papillomas Diagnosed on Core Biopsy}

Salik Nazeer, Mark T. Lawton, Joseph J. Weber, Franklin R. Zuehl

Departments of Radiology, Surgery, and Pathology, Aurora Health Care

Background: Solitary intraductal papillomas are benign, proliferative, mass-forming lesions arising in the lactiferous ductal system of the breast. Solitary intraductal papillomas are encountered with core biopsy of the breast, with an incidence of approximately 5\%. Although papillomas are considered benign, the lesions frequently are surgically excised after diagnosis on core biopsy. Excision has historically been performed due to a certain percentage of core biopsy cases being reclassified (upstaged) to atypia or malignancy based on pathologic results of excisional biopsy.

Purpose: The objective of this study is to determine the incidence of solitary intraductal papillomas that were subsequently reclassified to atypia or malignancy following complete surgical excision.

Methods: A retrospective analysis was performed that included review of pathology results and other data for intraductal papillomas diagnosed by core biopsy from 2010 through 2016 at participating Milwaukee-metropolitan Aurora Health Care facilities. Female patients with documented personal history of breast carcinoma prior to the core biopsy procedure and patients under the age of 18 were excluded from the study. Reports were reviewed by 4 different investigators to identify subsequent excisional biopsy results as well as follow-up radiology to confirm diagnoses. All data were reported using descriptive statistics.

Results: Our initial review included 390 patients who had the diagnosis of papilloma from a core biopsy. Of those, 154 patients did not undergo excisional biopsy (39\%). Of the 236 patients who had excisional biopsies, 203 patients had benign specimens (86\%), 15 had atypia with intraductal papilloma (6\%), 11 had adjacent atypia $(5 \%)$, and 7 had malignancy (3\%). The 7 patients with malignancy were reevaluated radiographically to assess for concordance of the core biopsy and excisional biopsy results. Of the 7,3 patients with core biopsy-proven papilloma demonstrated malignancy within the existing lesion (1\%) on subsequent excisional biopsy, and 4 patients were excluded due to lack of concordance between radiology and pathology findings comparing core biopsy location to excisional biopsy position.

Conclusion: In our facilities, only $1 \%$ of patients with initially diagnosed benign breast papillomas at core biopsy demonstrated true associated malignancy on the excisional biopsy specimen, a significantly lower rate than reported incidences in most similar previous studies. Our study is limited by our sample size. Further prospective studies would be helpful to further validate this data. 


\section{Specialty Access for the Uninsured Program: System Costs Before and After Enrollment}

Sally Smaida, Widalys Calderon Torres, Jessica J. F. Kram, Steve Ohly, Dennis J. Baumgardner

Aurora Walker's Point Community Clinic; Department of Family Medicine, Aurora UW Medical Group; Center for Urban Population Health

Background: The Specialty Access for Uninsured Program (SAUP) is a partnership within Milwaukee County in which safety-net primary care clinics are paired with hospital/health systems for specialty care. Health systems are assigned a portion of the income-eligible uninsured patients in the county, who then receive specialty services at no cost.

Purpose: To examine differences in cost, hospitalization, and use of emergency department (ED) and urgent care prior to and following SAUP enrollment.

Methods: We prospectively identified and retrospectively reviewed patients, age $\geq 18$ years, residing in Milwaukee County who were enrolled in SAUP during 2017. Several variables were retrospectively collected prior to and following initial SAUP enrollment. Calendar-year cost data were identified per patient for the year prior to SAUP enrollment (2016), the year of SAUP enrollment (2017), and the year following SAUP enrollment (2018). Costs in 2016 and 2018 were adjusted to match 2017 costs. Paired t-tests were used to compare care (eg, number of hospital admits 12 months prior to and following SAUP enrollment) and cost-related differences. A P-value less than 0.05 was considered significant.

Results: Of the original 99 patients enrolled in SAUP, 13 were excluded for not following up with care. Previously reported patient characteristics remained similar. Only $67.4 \%$ of SAUP patients were established patients 12 months prior to SAUP enrollment; $73.3 \%$ were established patients 6 months prior to enrollment. Mean time between enrollment and specialty service visit was 30.8 days (median: 25.0 days), and $46.5 \%$ had contact with a primary care provider following specialty services. Mean number of hospital admissions (0.14 vs 0.15), ED admissions (0.36 vs 0.30), and urgent care visits (0.02 vs 0.00$) 12$ months prior to and following SAUP enrollment, respectively, were not statistically different. However, the mean number of no-show visits 12 months prior to and following SAUP enrollment declined significantly ( 0.89 vs 0.34 , respectively; $\mathrm{P}=0.001)$. Mean charges significantly increased from 2016 to 2017 ( $\$ 7048$ vs $\$ 35,091$, respectively; $\mathrm{P}=0.002$ ) and from 2016 to 2018 ( $\$ 7048$ vs $\$ 14,552$, respectively; $\mathrm{P}=0.034$ ), but significantly decreased from 2017 to 2018 ( $\$ 35,091$ vs $\$ 14,552$, respectively; $\mathrm{P}=0.013$ ).

Conclusion: Charge increases during the years of specialty episode care are reflective of care delivered. A significant decline in the number of no-show visits indicates the possible development of a clinic relationship, often seen when patients establish trust in a medical home.

\section{Cryptococcal Antigen Testing in an Integrated Medical System: Eastern Wisconsin}

Marianne Klumph, Brian Hoeynck, Dennis J. Baumgardner

Department of Family Medicine, Aurora UW Medical Group; Center for Urban Population Health; Aurora Research Institute
Background: Cryptococcosis is an environmentally acquired endemic fungal infection causing serious disease in usually immunocompromised hosts. Environmental associations of pathogenic species include certain trees, soils, bird guano, and parks. Little is known about the frequency and distribution of cryptococcosis in Wisconsin. Cryptococcal antigen detection (CrAg) test is more than $90 \%$ sensitive and specific and is frequently used to screen patients with likely disease.

Purpose: We aimed to explore the geodemographic and clinical features of Wisconsin patients tested with $\mathrm{CrAg}$.

Methods: We conducted a retrospective review of all patients at Aurora Health Care having CrAg testing from January 2013 to April 2017. Patients with 1 or more positive CrAg test(s) were always considered positive, and basic demographic and clinical features were used from the first identified positive test. For patients always testing negative, clinicodemographic features were taken from the first test. Basic descriptive statistics were computed and chi-squared or t-tests were used as appropriate. Binary logistic regression was used for multivariable analysis.

Results: A total of $1465 \mathrm{CrAg}$ tests (741 on serum, 723 on cerebrospinal fluid, 1 other) were performed on 1211 unique patients (mean age: $53.7 \pm 16.5$; female sex: $50.2 \%$; white race: $73.9 \%$ ) during this time. At least $1 \mathrm{CrAg}$ test was positive in 23 of 1211 patients $(1.9 \%)$; 21 of 23 (6 transplant patients, $5 \mathrm{HIV}, 4$ malignancy, 6 other) were immunocompromised (vs 13 predicted; $\mathrm{P}<0.001$ ). Positive patients were disproportionately male ( 19 of 23 [3.2\% of all those tested] vs 4 female $[0.7 \%$ of all those tested]; $\mathrm{P}=0.002)$ and nonwhite ( 12 of 23 [3.8\% of all those tested] vs 11 white $[1.2 \%$ of all those tested]; $\mathrm{P}=0.012$ ). These associations, but not age, were significant independent predictors in multivariable models. Overall, 17 separate zip codes had 1 positive case, 2 city of Milwaukee zip codes had 3 each, with the closest two patients living 193 meters apart; no other case clustering or close proximity to waterways was observed ( $41 \%$ were less than $162 \mathrm{~m}$ from green space, similar to historical controls). Positive patients were more prevalent in Milwaukee $(2.9 \%$ of those tested vs $1.6 \%$ of all those tested in rest of state), but this was not significant ( $\mathrm{P}=0.21)$.

Conclusion: Among all CrAg-tested patients, male gender, nonwhite race/ethnicity, and immunocompromised status, not natural environmental features, predicted positive tests. To our knowledge, this is the first report regarding the distribution of cryptococcosis in Wisconsin.

Hot Spotting Medically Complex At-Risk Patients: Does It Decrease Hospital and Emergency Department Admissions?

Melissa Grube, Glenda Sundberg, Dane Olsen, Jessica J.F. Kram

Department of Family Medicine, Aurora Health Care; Aurora St. Luke's Family Practice Center; Aurora UW Medical Group; Center for Urban Population Health

Background: Our previous research has shown that hot spotting medically complex at-risk patients may improve patient outcomes and decrease health care costs.

Purpose: The purpose of this project was to extend our previous efforts for the most complex patients at the Family Practice Center clinic in Aurora St. Luke's Medical Center and add one additional site, the Family Care Center clinic at Aurora Sinai Medical Center. 
Methods: During 2018, new at-risk patients were identified and tracked at two family medicine sites. Each patient verbally consented to voluntary participation in the hot spotting intervention. A new interdisciplinary team was created at the second clinic site, which required additional buy-in to the intervention's concepts. We compared number of hospital admissions, emergency department (ED) visits, and estimated cost savings before and after a 9-month intervention period. Basic descriptive statistics were conducted to describe the overall population. Paired t-tests were used as appropriate. A P-value less than 0.05 was associated with statistical significance.

Results: A total of 35 new "hot spot" patients were identified. This patient cohort had mean age of 56 years and was predominately female (69\%). Prevalence of mental illness was $89 \%$. Additionally, $66 \%$ had communication problems $(17 \%$ with primary language other than English), 63\% had caregivers suffering from caregiver fatigue, and $49 \%$ had safety concerns. Furthermore, $34 \%$ of patients were considered underutilizers of health care. Although not statistically significant, mean total ED visits (3.6 vs $2.7 ; \mathrm{P}=0.102)$ and hospital admissions (1.3 vs $0.8 ; \mathrm{P}=0.087)$ decreased per person. Charlson scores did not change following the intervention. Cost reduction based on these decreased rates was determined at $\$ 87,000$ ( $\$ 2485 /$ patient), equating to a 2.09 return on investment. Even without knowing the financial aspects, clinic staff were very satisfied with the intervention. Overall, 97\% of provider respondents felt hot spotting patients was a good idea after the intervention, compared to $78 \%$ before, and $76 \%$ felt the intervention helped them take better care of their patients.

Conclusion: Extending our hot spotting intervention to an additional clinic site showed similar decreases in hospital and ED utilization as previously reported. Moreover, there continued to be cost savings at both sites. Therefore, our pilot efforts will be extended to a third nonresidency clinic site in 2019 for further evaluation.

\section{Reduced Esophageal Cooling With Left Atrial Roof and Posterior Wall Cryoablation}

Dhruv Chawla, Lynn Erickson, Mohamed Djelmami-Hani, Imran Niazi

\section{Aurora Cardiovascular Services; Aurora Research Institute}

Background: Thermal esophageal injury is a rare but devastating complication of pulmonary vein isolation using radiofrequency energy or cryoablation (CRYO) and also radiofrequency ablation of posterior wall (PW). Risk of esophageal injury with PW and roof CRYO is unknown. Since PW and roof CRYO has recently been advocated, we studied minimum esophageal temperature (MET) to assess safety of PW and roof CRYO.

Purpose: To assess safety of PW and roof CRYO for atrial fibrillation.

Methods: CRYO was performed in 16 patients (mean age: 64 \pm 9 years; mean left atrial volume: $59 \pm 24 \mathrm{~mL} / \mathrm{m}^{2}$; male sex: 12) with atrial fibrillation ( 8 paroxysmal, 8 persistent). Following pulmonary vein isolation, CRYO balloon was dragged across the roof and PW with lesions delivered at half-balloon-width intervals. CRYO balloon and esophagus were precisely localized to $1 \mathrm{~mm}$ accuracy with Navik 3D mapping system (APN Health, LLC, Waukesha, WI). Lesions were maintained for 3 minutes unless a MET of $<28^{\circ} \mathrm{C}$ was encountered. Incidence of lesions with a MET of $28^{\circ} \mathrm{C}$ was compared between pulmonary veins, PW, and roof sites using Fisher's exact test.

Results: Lesions were distributed as follows: roof ( $n=81)$, PW $(n=34)$, and pulmonary veins (right superior [RSPV, $n=19$ ], right inferior [RIPV, $n=21$ ], left superior [LSPV, $n=19$ ], and left inferior [LIPV, n=17]). Occurrence of MET of $\angle 28^{\circ} \mathrm{C}$ was: 1 of 81 roof, 2 of 34 PW, 2 of 21 RIPV, 2 of 17 LIPV, and no RSPV/LSPV lesions. MET of $<28^{\circ} \mathrm{C}$ was more common in RIPV $(9.5 \%)$ and LIPV (11.8\%) when compared to roof (1.2\%) and PW (5.9\%); these differences were statistically significant $(\mathrm{P}<0.001$ for all comparisons).

Conclusion: Significant esophageal cooling with CRYO is less common at the roof and PW than in inferior pulmonary veins, and the overall incidence is low. CRYO of PW and roof may be safer than CRYO of pulmonary veins.

\section{Goals of Care Conversations: An Evaluation}

Elizabeth A. Duthie, Colleen M. Galambos, Theresa Frederick, Jacob Verkuilen, Tim Jessick

Center for Urban Population Health; Helen Bader School of Social Welfare, University of Wisconsin-Milwaukee; Continuing Professional Development, Aurora Health Care; Palliative Medicine, Aurora Health Care

Background: Growing evidence suggests that physician and patient discussions about goals of care improve quality of life, quality of dying, and decrease end-of-life intensity of care. However, these conversations do not occur at the frequency desired by seriously ill and dying patients. Practitioners recommend new approaches to increase the frequency and quality of such conversations. The "Teaching Primary Palliative Care" training offered through Aurora Health Care's continuing professional development office is one new approach aimed to equip physicians with skills to increase the frequency and quality of goals of care conversations.

Purpose: To evaluate the Teaching Primary Palliative Care (TPPC) training to determine if physicians report increased comfort in engaging in goals of care conversations with seriously ill and dying patients posttraining.

Methods: TPPC training occurs in three stages. Stage A: Initial self-assessment completed online. Participants complete a survey in which they answer a series of questions designed to measure comfort level in engaging in goals of care communications with their seriously ill and dying patients. Stage B: Attend an in-person training session on communication skills and techniques on having goals of care conversations with patients. Stage C: Second self-assessment completed online within about 3 months after physicians practiced and applied skills in their clinical practice. In fall 2018, 78 Aurora physicians completed stages A and B of the training, resulting in 37 completed stage $A$ and stage $C$ selfassessments. To assess for increased comfort level, the stage A and stage $\mathrm{C}$ assessments were statistically analyzed using the 1 -sided sign test.

Results: Compared to their reported comfort level prior to completing TPPC training, physicians' comfort level after training increased in 4 out of 5 circumstances related to engaging in goals of care conversations with patients: discussing CPR/do not resuscitate $(\mathrm{P}=0.018)$; discussing hospice or palliative care referral $(\mathrm{P}<0.001)$; discussing artificial hydration or nutrition $(\mathrm{P}=0.001)$; and discussing prognosis specifics $(\mathrm{P}=0.002)$. 
Conclusion: Findings suggest that TPPC training increases the comfort level of physicians to engage in goals of care conversations with seriously ill and dying patients. Next steps for this project include a longitudinal study to determine whether physicians continue to be more comfortable in these circumstances 6-9 months after completing the training.

\section{Effectiveness of SBIRT Training Program for Advanced- Standing Master of Social Work Students}

\section{Elizabeth Miecielica, Lisa Berger}

Helen Bader School of Social Welfare, University of Wisconsin-Milwaukee; Center for Urban Population Health

Background: Screening, Brief Intervention, and Referral to Treatment (SBIRT) for substance misuse is an evidence-based, public health approach for early identification and intervention of risky alcohol and other substance use. Recent SBIRT dissemination efforts have included training grants to medical schools and university programs of nursing and social work. These efforts come at a time of greater focus by higher education accreditation bodies on competency-based education.

Purpose: The purpose of this study was to examine the effectiveness of a SBIRT training program for advanced-standing Master of Social Work students. The objective was to develop a program that would train students to competency in delivering an evidence-based SBIRT protocol.

Methods: A total of 91 students participated voluntarily in the study. All students received the same SBIRT training, consisting of an initial motivational interviewing module ( 8 hours) followed by the SBIRT protocol module (4 hours) conducted within a course on substance misuse interventions. Competency was assessed by a modified version of a valid brief intervention adherence scale as used by two trained raters. Raters independently listened to an audiotaped SBIRT protocol assignment submitted by each participant. Students achieved competency if at least $80 \%$ of the SBIRT protocol elements were deemed executed by both raters. Fidelity to each of the 4 SBIRT protocol subsections of engaging, focusing, eliciting, and planning also was examined.

Results: The overall average interrater reliability as assessed by proportion of agreement was 0.82 , or moderate agreement $(0.90=$ high agreement). In total, 75 of the 91 students $(82 \%)$ achieved competency, while fidelity to each of the SBIRT protocol subsections was as follows: engaging $96 \%$; focusing $92 \%$; eliciting $46 \%$; and planning $56 \%$. For students who did not achieve competency $(\mathrm{n}=16$, or $18 \%)$, most did not deliver to fidelity the eliciting (94\%) and planning (88\%) subsections.

Conclusion: The SBIRT training program was effective in training to competency 4 out of 5 Master of Social Work students. The vast majority of students, regardless of overall competency, delivered the engaging and focusing subsections with fidelity. Regardless of overall competency, most students tended to miss elements in the planning subsection, while students who did not achieve competency tended to also miss elements in the eliciting subsection, indicating more time and/or revised training strategies are needed to increase fidelity and, thus, competency.
Evaluation of the Association Between Thrombotic and Bleeding Outcomes and Bridging Anticoagulation Strategies in Patients With Left Ventricular Assist Device

Marian Dobles, Sarah Zukkoor Zorn, Jon Godden, Rachel Pedersen, Vinay Thohan

Department of Pharmacy, Aurora Health Care; Tendick Center for Advanced Heart Failure Therapies

Background: In patients with a left ventricular assist devices (LVAD), long-term anticoagulation with a warfarin regimen is required to prevent thromboembolic events, including device thrombosis. Patients with LVAD frequently experience subtherapeutic international normalized ratio (INR) and/or interruption of anticoagulation. In these cases, bridge therapy should be considered, taking into account the balance between thrombotic and bleeding risk. Bridging strategies include anticoagulation with intravenous heparin and low-molecularweight heparin.

Purpose: To assist in the expansion of anticoagulation approaches for the LVAD population.

Methods: This is a retrospective, observational, single-center study of patients with LVAD. Patients were identified through the Interagency Registry for Mechanically Assisted Circulatory Support (INTERMACS) database. Patients implanted from January 2012 to December 2017 and who survived 30 days posthospitalization were followed until time of explant, transplant, or death. A bridging event is defined as the start of anticoagulation until 30 days post. Primary outcomes include thrombotic events and major bleeding events as reported in the INTERMACS registry. A generalized estimating equations model was used to analyze associations between baseline and temporal predictors of thrombotic and major bleeding events.

Results: A total of 156 patients were included. During followup time, 109 major bleeding events occurred, with 30 unique bridging events utilizing enoxaparin and 227 utilizing heparin. We adjusted for baseline predictors associated with major bleeding events. Therapeutic INR per protocol was associated with reduced risk of bleeding (relative risk [RR]: 0.47 [0.260.87]; $\mathrm{P}=0.02$ ). Compared to no bridging, patients bridged on heparin experienced significantly fewer bleeding events (RR: $0.43[0.21-0.87] ; \mathrm{P}=0.02)$. There was no difference between patients bridged on enoxaparin compared to no bridging (RR: $0.91[0.34-2.34] ; \mathrm{P}=0.85)$. Results on thrombotic events are pending.

Conclusion: Compared to patients who were not bridged, patients bridged with enoxaparin had no increase in bleeding events, whereas patients bridged with heparin experienced lower rates of bleeding. Although known bleeding risk factors were adjusted for, these results may be limited by physician discretion of bridging based on individual patient risk. Further data analysis will be conducted to identify independent bleeding and thrombotic risk factors to assist in anticoagulation practices in patients with LVAD. 


\section{Epidemiology of Male Breast Cancer}

Santhi D. Konduri, Maharaj Singh, George C. Bobustuc, Richard A. Rovin, Amin B. Kassam

\section{Aurora Research Institute; Neuro-Oncology Research, Aurora Health Care; Aurora Neuroscience Innovation Institute}

Background: Male breast cancer is relatively rare (less than $1 \%$ of all cases of breast cancer) and usually treated similarly to female breast cancer. There are a very limited number of studies for characterization of male breast cancer, and most studies are small and underpowered. We report the results of a retrospective study using national cancer database from years 2004 to 2014 .

Purpose: The purpose of this study was to determine the survival and risk factors for male breast cancer in using a large study population in the United States.

Methods: In this retrospective study we included 19,795 patients with a follow-up period of up to 11 years. In this population, the majority of patients were non-Hispanic white $(74.8 \%), 12.1 \%$ were black, $0.5 \%$ were Hispanic, and the remaining were other races.

Results: The rate of incidence was $7.2 \%$ in 2004 and increased to $10.3 \%$ in 2014 . The significant predictors of mortality included cancer stage II, III, and IV vs stage 0 (hazard ratio [HR]: 1.5, 2.7, and 9.9, respectively), age (HR: 1.04), surgery (HR: 0.4), radiation (HR: 0.8), chemotherapy (HR: 0.8), hormonal (HR: 0.8), tumor size for every 10-mm increase (HR: 1.01), and estrogen receptor (ER)-positive and progesterone receptor (PR)-positive (HR: 0.8). Socioeconomic factors such as income, insurance, and facility type also contributed to the higher risk for mortality. Higher CharlsonDayo index resulted in higher mortality rate. Kaplan-Meier survival probability was higher in patients who underwent surgery $(\mathrm{P}<0.001)$, received radiation $(\mathrm{P}<0.001)$, received chemotherapy $(\mathrm{P}<0.001)$, or received hormonal therapy $(\mathrm{P}=0.0126)$. The patients identified with ER and PR positivity $(\mathrm{P}<0.0001)$ also had higher survival probability. In a subset of patients $(n=7880)$, HER2/neu was available from 2010 to 2014). Survival probability was higher in patients with negative HER2/neu expression $(\mathrm{P}<0.001)$, whereas mortality risk was 1.7 times higher among patients diagnosed with positive HER2/neu expression.

Conclusion: This study with a large patient population showed that socioeconomic factors, cancer stage, tumor characteristics (size and grade), and higher Charlson-Dayo scores contributed to higher mortality among male patients diagnosed with breast cancer. Among treatment modalities, surgery was most effective followed by radiation, chemo-, and hormonal therapy. Patients identified with positive ER and PR had better survival probability. Biomarker identification followed by timely diagnosis of the treatment, irrespective of socioeconomic status, is likely to reduce the risk for mortality. A subset analysis showed that positive HER2/neu expression had higher mortality.

\section{Cardiac Catheterization and Acute Ischemic Stroke: Who Is at Risk?}

Maharaj Singh, Reji Babygirija, Richard A. Rovin, Kessarin Panichpisal

\section{Aurora Research Institute; Aurora Neuroscience Innovation Institute}

Background: Cardiac catherization is a well-established invasive procedure that is used to diagnose and treat coronary artery disease. Estimated annual rates for cardiac catherization range from 171 to 650 per 100,000 population ( $>20$ years of age) for both sexes. Cardiac catherization has been associated with an increased risk of acute ischemic stroke. Timely and specialized treatment of ischemic stroke is crucial for increasing survival and reducing morbidity.

Purpose: Identify predictors of acute ischemic stroke in patients undergoing cardiac catherization procedure.

Methods: Data on patients who underwent cardiac catherization from January 2008 to March 2017 was retrospectively collected and analyzed. Demographics and comorbidities were compared among those patients with and without incident stroke using area under the curve c-statistic.

Results: A total of 35,268 patients were included in the study. Of these, $59 \%$ were male, $87.5 \%$ were white, $10.7 \%$ were black, and mean age was $66.1 \pm 12.7$ years. Acute ischemic stroke was reported among only $0.4 \%$ of these patients. The main predictors for acute ischemic stroke, based on a c-statistic of 0.68 , were age (in 10-year increment) at the time of procedure (adjusted odds ratio [aOR]: 1.37, 95\% CI: 1.10-1.63; P<0.001), atrial fibrillation (aOR: 2.36, 95\% CI: 1.16-4.80; $\mathrm{P}=0.0182$ ), hypertension (aOR: 0.53 , 95\% CI: $0.34-0.82 ; \mathrm{P}=0.0046$ ), and history of stroke (aOR: 3.15 , 95\% CI: $1.94-5.11 ; \mathrm{P}<0.001)$.

Conclusion: Retrospective assessment of nearly 10 years of cardiac catherization procedures in a single center revealed that incident acute ischemic stroke is a relatively rare occurrence. The independent predictors for stroke were age, atrial fibrillation, and history of prior stroke. Importantly, hypertension was found to be protective of acute ischemic stroke; however, data on evidencebased therapy (such as beta blocker, statin, or antiplatelet use) were not analyzed due to missing values for more than two-thirds of the patients. In the future we will strive to develop a risk score for predicting acute ischemic stroke among the patient population undergoing cardiac catherization.

\section{Evaluation of Sox2 Expression in Glioblastoma Stem Cells During Zika Virus Infection}

Beau Adams, Sam D. Zwernik, Amin B. Kassam, Richard A. Rovin, Parvez Akhtar

Aurora Research Institute; Aurora Neuroscience Innovation Institute

Background: Glioblastoma is a deadly form of brain cancer. Standard treatment involves surgery followed by chemotherapy and radiation. Most tumors recur within 6 months, fueled by a small population of glioblastoma stem cells (GSCs) that resist and survive treatment. There are no pharmacological agents currently available for specific targeting of GSCs. Zika virus (ZIKV) is a flavivirus and is linked to congenital microcephaly in addition to other neurological manifestations. It appears that ZIKV selectively targets normal neural stem cells in the developing brain, causing the microcephaly. The specificity is due to receptor-mediated cell entry: a tyrosine kinase receptor, AXL, present on neural stem cells, specifically binds ZIKV and permits cell entry. We examined the effect of ZIKV on Sox2, a transcription factor that controls the self-renewal of stem cells.

Purpose: The purpose of this study is to understand the role of Sox2 in ZIKV infection in glioblastoma cancer stem cells. 
Methods: ZIKV strain MR766 was propagated in Vero cells. Viral stock was titrated by plaque assays. Glioblastoma patientderived cell lines were infected with ZIKV, and the percentage of infected cells was quantified by flow cytometry using a pan-flavivirus antibody. Western blot was used to characterize Sox 2 expression in 5 glioblastoma patient-derived cell lines. Immunofluorescence microscopy was used to visualize Sox2 levels after ZIKV infection.

Results: We found that Sox2 expression varies among the glioblastoma patient-derived cell lines (7754, 7730, 7714, 7753, and 7978) and is inversely correlated with AXL expression. We further found that ZIKV infection inhibits Sox2 levels in a dosedependent manner in all 5 glioblastoma cell lines.

Conclusion: Our results suggest that ZIKV infection causes loss of self-renewal through Sox 2 in glioblastoma cancer stem cells. 